\title{
News from the IAEH
}

\section{Discussion on the Role of National Public Health Agencies in the Implementation of Ecohealth Strategies for Infectious Disease Prevention}

Why should ecohealth principles be adopted for infectious disease control by public health agencies in high-income countries? Infectious diseases (IDs) remain the most important contributor to all-ages mortality in low- and middle-income (LMI) countries. Although high-income countries face more substantial burdens from chronic diseases, emerging infections (e.g., West Nile virus, HIV, SARS, avian influenza, etc.) and outbreaks of foodborne pathogens are reminders that advanced wealth, sanitation, and technology do not offer complete protection against IDs.

Consistent with these realities, the U.S. Centers for Disease Control and Prevention (CDC) and the Public Health Agency of Canada (PHAC) have for decades supported ID research, surveillance, vaccination campaigns, and outbreak responses. These agencies have been highly successful at reducing the ID burden in the United States, Canada, and abroad. However, the spread of antibiotic resistance, global pandemics of emerging IDs, increased ID incidence linked to climate change and biodiversity loss (e.g., Lyme disease and hantavirus), and difficulties in vaccine development for IDs (e.g., for HIV and the dengue

Published online: May 18, 2013

Correspondence to: Karlyn Beer, e-mail: Kdb26@uw.edu virus) suggest that a new parallel approach focused on prevention is necessary.

Such an alternative approach can be found in the young scientific disciplines of Ecohealth and Conservation Medicine, as well as the One Health Initiative, collectively referred to here as "ecohealth." Each of these fields, despite three different names, recognize human and animal diseases as emergent properties of a complex system and thus integrate many disciplinary factors into ID research, including ecology, biology, land use, demographics, travel, social inequalities, and climate. Already, this "systems approach" has demonstrated causal associations between ocean chemistry and cholera incidence and between landuse and climate change and numerous zoonoses, such as Lyme disease and dengue fever. In the United States, landuse changes affect Lyme disease reservoir abundance and diversity, which in turn influence infection prevalence and disease risk. For dengue fever, models of climate-driven changes in mosquito vector abundance have been recapitulated in annually reported cases in Central America. These ecohealth-based insights offer vast opportunities for novel and complementary prevention strategies.

The prospect of an effective systems approach to ID prevention is compelling, especially for students interested in public health practice in the United States and Canada. Yet, why do public agencies in high-income countries seem to play a minimal role in strategic ecohealth implementation? The role that major U.S. and Canadian public health agencies should play in ecohealth project leadership seems unclear. For example, the CDC has an Office of One Health within the Division of High-Consequence Pathogens and 
Pathology. Its mission is aligned with ecohealth principles, but documentation of related projects is scarce. Likewise, the PHAC hosted a "One World, One Health" conference in Winnipeg in 2009, where experts convened to discuss how to implement an ecohealth approach to ID surveillance, monitoring, and prevention. However, information on follow-up to projects that may have emerged from this meeting is not readily available.

Who is leading the implementation of ecohealth-based ID prevention? The Canadian International Development Research Center (IDRC), which funds ecohealth research, recently published an analysis of 15 applied ecohealth case studies. These projects were most often initiated by researchers in LMI countries with heavy ID burdens. Many projects achieved success through local government support and did not cite outside agency involvement.

There are many potential explanations for the observation that the well-equipped health agencies in the United States and Canada have seemed slow to participate in ecohealth implementation. First, the ecohealth approach is young. While quality research has been done, there is still much to do, and application generally follows after a scientific field matures. Second, despite strong infrastructure, large public health agencies are often bureaucratic and slower to adopt new approaches and paradigms than are academia or the nonprofit sector. Third, IDs that lend themselves to an ecohealth approach are larger problems in LMI countries, and thus there is greater urgency for pioneers in these localities to implement ecohealth-based research on their own. Nonetheless, it is possible that the CDC and PHAC are playing a greater role than their websites and publications reveal, and these efforts may or may not carry the "ecohealth" label. Perhaps a central repository, or a concerted effort to publicize ecohealthrelated projects, would better represent current and ongoing cross-disciplinary efforts at the CDC and PHAC.

What should be the role of U.S. and Canadian public health agencies in strategic ecohealth implementation for ID prevention? One broad suggestion comes from the environmental microbiologist and former NSF director Dr. Rita Colwell, who has called for interdisciplinary "Centers of Ecological Health" that will bring all stakeholders together, including national public health agencies, to reduce global ID burdens. Toward this end, what are the CDC and PHAC doing to make ecohealth strategies like this a reality, and what should the next steps be? How can students stay better informed of agencies' ecohealthfocused activities, and how might students shape their training to be involved in what is certainly an important new direction for public health?

From a student perspective, it is exciting and encouraging to see the recent uptick in ecohealth-specific graduate programs and post-graduate fellowships. Nevertheless, it can be challenging to find a place in public health where ecohealth approaches and skills may be applied. For example, health agencies often have separate divisions for infectious disease and animal health, and agencies involved in land use and planning are separate from public health altogether. What positions are available to graduates interested in coordinating among these divisions? Perhaps this challenge reflects the excitement and chaos of a nascent paradigm shift in infectious disease prevention. At this point, students cannot expect to find job descriptions that match the forward-thinking, interdisciplinary work we aim to do. Instead, students themselves are part of the paradigm shift and, if we are persistent and innovative, we may carve out the necessary niche for ecohealth within public health. If we are successful, we will be the ones writing the requests for applications, not seeking them.

\section{Karlyn D. Beer}

Interdisciplinary graduate program in Molecular and Cellular Biology and Epidemiology, Institute for Systems Biology and University of Washington, Seattle, WA

\section{Professional Responses}

Public Health Agencies and Ecohealth Strategies: Opportunity Unanswered?

As an ecologist who spent 25 years working in public health, including 16 years at CDC, I have some insights that span the EcoHealth spectrum. Ms. Beer is correct; CDC, and probably other public health agencies, have not fully and actively embraced the One Health/EcoHealth concept. Unlike academic investigators who have much autonomy, CDC researchers operate within a defined "mission," dictated by policies that are not independent of political pressures. Projects pursued are generally those that will yield clear, short-term results. Rushing teams of physicians and epidemiologists to stop an outbreak is important. It also makes headlines and gets people promoted. Working with residents to establish livelihoods that protect ecosystem integrity, maintain biodiversity, and prevent outbreaks from occurring is also important. Yet it goes unnoticed, produces no heroes, is unlikely to win votes or 
launch careers, and it may not secure continued agency funding.

Public health agencies are beginning to accept the OneHealth concept as a useful adjunct. Nevertheless, clear ideas of how to apply the concept have not been developed and agencies are unwilling to take money from traditional programs and invest in what is seen as a new and largely unproven approach. My experience at CDC has shown that change from within happens slowly. However, when strong direction comes from the top, change is rapid. Government agencies (including the $\mathrm{CDC}$ ) answer to Congress and the Administration, who, in turn, answer to the citizens.

What can students do? Obviously, seek collaborations with and jobs within public health agencies. Conduct research that demonstrates the effectiveness and impact of One-Health approaches. Importantly, this generation of scientists must not repeat the mistakes of the past and sequester itself within the ivory tower. Remain involved with local communities, regain the public trust, share knowledge and exchange ideas with the community, and educate our congressional representatives.

\section{James N. Mills}

Population Biology, Ecology and Evolution Program, Emory University, Atlanta, GA; Viral Special Pathogens Branch, National Center for Emerging and Zoonotic Infectious Diseases, Centers for Disease Control and Prevention, Atlanta, GA

\section{Change in Culture}

The profile of the One Health (or ecohealth) movement has come increasingly to the forefront in discussions of emerging IDs. Given the complex interplay of factors that affect emergent zoonotic diseases (witness such high profile infectious disease events of the past decade as SARS, swineorigin H1N1, monkeypox, and peanut butter salmonellosis), this trend is entirely justified and indeed overdue. How have governmental public health agencies responded? The bureaucratic response to create additional, often siloed entities, may indeed not be the most productive one, as Ms. Beer notes. Also, in an era of shrinking budgets and government stretched thin, we are not likely to see real progress in the creation of new capacity in the near term. Instead, people within existing agencies need to understand the need for this new capacity, and feel sufficiently empowered to reach across agency boundaries to the resources they need.
This in turn requires a change in culture that is in many ways more difficult to affect than merely creating a new organizational structure. Veterinary health, human health, plant health and ecology occupy very different spheres not only in government. These disciplines are taught in different schools, published in distinct journals, discussed at divergent conferences. The one health movement is, gradually, eroding boundaries and bringing these disciplines to the same table. The establishment of journals like EcoHealth, the creation of meetings like One World, One Health and the International Meeting on Emerging Diseases, the endorsement of the movement by professional organizations like the AMA and AVMA, the growth of surveillance systems like ProMED that monitor plant, animal, and human health, and even academic programs like Ms. Beer's speak to this change. We are increasingly seeing this transformation within governmental organizations as well, perhaps not leading the cultural shift, but certainly taking part in it.

\section{Lawrence C. Madoff}

Division of Infectious Diseases and Immunology, University of Massachusetts Medical School, Worcester, MA; Division of Epidemiology and Immunization, Massachusetts Department of Public Health, Boston, MA

\section{A Vector-Borne Disease Perspective}

Many IDs have critical environmental links. Consequently, changing ecologies can influence disease emergence. A prime example is Lyme disease emergence and the links to reforestation, habitat fragmentation, and decreasing species biodiversity. The One Health initiative promotes the fact that disease emergence occurs at the interface of humans, other animals, and a changing environment.

Despite articulation, the One Health concept struggles for a place in mainstream public health. In part, this may be due to the reality that public health is much broader than it used to be, and any single priority competes with many others. When the U.S Centers for Disease Control and Prevention (CDC) was established in 1946 as the Communicable Diseases Center, IDs were the sole focus, and ecohealth, while not birthed as a term, was the lens through which entomologists, engineers, and epidemiologists studied and evaluated interventions for diseases like malaria, plague, and polio. Today, however, public health at all 
levels is pressed by economic constraints and a vastly expanded mission.

Regardless of the challenges, One Health is very much alive within the world of public health. It is seen in the ArboNET surveillance system that maps data on arboviruses detected in humans, birds, mosquitoes, and livestock. It is seen in Lyme disease projects that evaluate upstream interventions such as reservoir-targeted vaccines. It is further demonstrated in novel interdisciplinary training programs such as the one that provides cross-training in both public health and climatology and atmospheric sciences, for the purpose of developing young scientists to provide leadership in addressing the challenges that climate disruption and change will bring to the world of public health.
While these programs are small and seminal, they indicate a growing recognition of the importance of a systems approach to public health, one that demonstrates an evolving ecohealth perspective.

Charles B. Beard

Division of Vector-Borne Diseases, National Center for Emerging and Zoonotic IDs, Centers for Disease Control and Prevention, Fort Collins, CO

Carol Rubin

One Health Office, National Center for Emerging and Zoonotic IDs, Centers for Disease Control and Prevention, Atlanta, GA 